\title{
A clinical pathway for safely and effectively cardioverting emergency department patients with atrial fibrillation greater than 48 hours
}

\author{
Baha Zaro ${ }^{1}$, Evan Avraham Alpert ${ }^{1}$, Nechama Kaufman ${ }^{1}$, and David Rosenmann ${ }^{1}$ \\ ${ }^{1}$ Shaare Zedek Medical Center
}

February 22, 2021

\begin{abstract}
Background: The current emergency medicine literature on cardioversion for atrial fibrillation (AF), describes its performance on those who are hemodynamically unstable, present within 48 hours of the onset of the arrhythmia, or are on long term anticoagulants. This article describes a clinical pathway comparing patients presenting to the emergency department (ED) with atrial fibrillation (AF) of more than 48 hours who underwent a transesophageal echocardiogram (TEE) and subsequent cardioversion in the ED. The objective of this study is to evaluate such a pathway looking at the time to cardioversion, length of hospital stay, rate of successful cardioversion, and the rate of complications compared to the traditional pathway of admitting patients directly to the cardiology department for evaluation and treatment. Methods: This was a retrospective observational study of patients who presented to the ED with AF for more than 48 hours, underwent a transesophageal echocardiogram, and then were electrically cardioverted either in the emergency department versus the cardiology ward. Results: Electrical cardioversion was performed in the ED on 92 patients (61\%) and the cardiology department on 59 (39\%). Over 90\% of cardioversions were successful in both groups. Time to cardioversion was significantly less in the ED group versus the cardiology group $(1.03 \pm 0.8$ days versus $4.17 \pm 1.9 ; \mathrm{p}<0.001)$. Similarly, the mean length of hospital stay was less for the ED group (1.5 \pm 1.5 days versus $7.2 \pm 3.5 ; \mathrm{p}<0.001)$. Conclusion: Patients who present in atrial fibrillation for more than 48 hours and then have a TEE, undergo electrical cardioversion faster in the ED compared to the cardiology ward. This clinical pathway also results in a shorter length of hospital stay without having more side effects.
\end{abstract}

A clinical pathway for safely and effectively cardioverting emergency department patients with atrial fibrillation greater than 48 hours

Baha Zaro, $\mathrm{MD}^{\mathrm{a}^{*}}$, Evan Avraham Alpert, $\mathrm{MD}^{\mathrm{a}^{*}}$, Nechama Kaufman, RN, MN ${ }^{\mathrm{b}}$, David Rosenmann, $\mathrm{MD}^{\mathrm{c}}$

1. Department of Emergency Medicine, Shaare Zedek Medical Center, Shmu'el Bait St 12, Jerusalem, Israel, 9103102

2. Department of Quality and Safety, Shaare Zedek Medical Center, Shmu'el Bait St 12, Jerusalem, Israel, 9103102

3. Jesselson Integrated Heart Centre, Shaare Zedek Medical Center, Shmu'el Bait St 12, Jerusalem, Israel, 9103102

*Equal contribution

Acknowledgements: The authors would like to acknowledge Emilya Zuty for her work contributing to maintaining the database used for the project.

Email Addresses:

Baha Zaro, MD: drbahazaro87@gmail.com 
Evan Avraham Alpert, MD: evanavrahamalpert@gmail.com

Nechama Kaufman, RN, MN: nechamak@szmc.org.il

David Rosenmann, MD: rosenmann@szmc.org.il

Grant support: none

Declarations of interest: none

Keywords: Transesophageal Echocardiography; Emergency Department; Electrical Cardioversion; Atrial Fibrillation; Cardiology Hospital Service

\section{Correspondence:}

Evan Avraham Alpert, MD

Shaare Zedek Medical Center

Shmu'el Bait St 12, Jerusalem, 9103102

Evanavrahamalpert@gmail.com

TEE for atrial fibrillation in the emergency department

A clinical pathway for safely and effectively cardioverting emergency department patients with atrial fibrillation greater than 48 hours

Background : The current emergency medicine literature on cardioversion for atrial fibrillation (AF), describes its performance on those who are hemodynamically unstable, present within 48 hours of the onset of the arrhythmia, or are on long term anticoagulants. This article describes a clinical pathway comparing patients presenting to the emergency department (ED) with atrial fibrillation (AF) of more than 48 hours who underwent a transesophageal echocardiogram (TEE) and subsequent cardioversion in the ED.

The objective of this study is to evaluate such a pathway looking at the time to cardioversion, length of hospital stay, rate of successful cardioversion, and the rate of complications compared to the traditional pathway of admitting patients directly to the cardiology department for evaluation and treatment.

Methods : This was a retrospective observational study of patients who presented to the ED with AF for more than 48 hours, underwent a transesophageal echocardiogram, and then were electrically cardioverted either in the emergency department versus the cardiology ward.

Results: Electrical cardioversion was performed in the ED on 92 patients (61\%) and the cardiology department on $59(39 \%)$. Over $90 \%$ of cardioversions were successful in both groups. Time to cardioversion was significantly less in the ED group versus the cardiology group (1.03 \pm 0.8 days versus $4.17 \pm 1.9 ; \mathrm{p}<0.001)$. Similarly, the mean length of hospital stay was less for the ED group ( $1.5 \pm 1.5$ days versus $7.2 \pm 3.5$; $\mathrm{p}<0.001)$.

Conclusion: Patients who present in atrial fibrillation for more than 48 hours and then have a TEE, undergo electrical cardioversion faster in the ED compared to the cardiology ward. This clinical pathway also results in a shorter length of hospital stay without having more side effects.

\section{What is already known on this topic?}

Atrial fibrillation is a common diagnosis in the emergency department. Patients who present with new-onset atrial fibrillation less than 48 hours or are on long term anticoagulation can be safely cardioverted in the emergency department. Patients who present with symptomatic atrial fibrillation longer than 48 hours and not on anticoagulation are traditionally admitted to the cardiology department for evaluation and treatment.

What does this article add? 
This article describes a clinical pathway whereby patients who present in atrial fibrillation for more than 48 hours and then have a transesophageal electrocardiogram showing no atrial clot, can return to the emergency department and safely undergo cardioversion. This clinical pathway also results in a faster time to cardioversion and shorter length of hospital stay compared to those admitted to the cardiology department.

Keywords: Transesophageal Echocardiography; Emergency Department; Electrical Cardioversion; Atrial Fibrillation; Cardiology Hospital Service

\section{Introduction}

Atrial fibrillation (AF) is the most frequently encountered dysrhythmia seen in the United States, both in the outpatient and emergency department (ED) settings. ${ }^{1}$ In 2014 in the United States, over half a million people visited EDs for AF, leading to a $62 \%$ admission rate and $\$ 10.1$ billion in adjusted annual charges for admitted patients. ${ }^{2}$

The treatment of patients presenting with AF is dependent upon several factors including the patient's hemodynamic status and the time of onset. If the patient presents when hemodynamically unstable, immediate conversion to sinus rhythm is required despite the time of onset of AF. For hemodynamically stable patients the issue then becomes rate and rhythm control. Rhythm control in the ED, whether by electrical or pharmacologic means is acceptable for those who have been anticoagulated for at least three weeks or if the atrial fibrillation is known to have started less than 48 hours. ${ }^{3}$ The "48-hour rule" that has been implemented in clinical practice is based on theoretical rather than evidence-based data. The risk of thromboembolism in patients undergoing cardioversion for AF of $<48$ hours duration is extremely low and ranges between $0-0.9 \%$. However, cardioversion of patients with AF $>48$ hours' duration without prior adequate anticoagulation led to a $5-7 \%$ risk of stroke thus leading to the 48 -hour rule. ${ }^{4}$ Cardioverting patients (electrically or pharmacologically) who present to the emergency department with AF of less than 48 hours' duration can be done safely and effectively by emergency physicians. ${ }^{5-8}$

Current guidelines for those with AF more than 48 hours and not on anticoagulation recommend either rate control and discharge from the ED on anticoagulation for at least 3 weeks before and at least 4 weeks after cardioversion, or performance of a transesophageal echocardiogram (TEE) with subsequent cardioversion if there is no clot on the atria or left atrial appendage. ${ }^{3}$

The objective of this study is to describe the safety and efficacy of a clinical pathway for patients who presented to the ED with $\mathrm{AF}>48$ hours, who underwent TEE, and then had synchronized electrical cardioversion performed in the ED versus the cardiology department. Major outcomes included time to cardioversion and length of hospital stay. Secondary outcomes included the rate of successful cardioversion, complications, and readmissions within 30 days of hospital discharge.

\section{Methods}

Study design and setting

This is a retrospective observational study of patients who presented to the ED between January 1, 2010, and December 31, 2018, with a diagnosis of AF for > 48 hours who then underwent a TEE. All of the TEEs were ordered from the ED, but the actual examination was performed by a board-certified cardiologist with additional training and extensive experience with this procedure. Of the patients who did not have an atrial clot or other contraindication to cardioversion, there was a convenience sample who returned to the ED for electrical cardioversion performed by emergency physicians versus a second group who were cardioverted in the cardiology department. Regardless of the place where electrical cardioversion was performed, all patients were sedated for the procedure and received anticoagulation both before and after their cardioversion as per protocol. The study site is a 1000-bed tertiary care medical center that includes a comprehensive cardiology department. Over 90,000 adult patients are seen annually in the ED. The Institutional Review Board waived the requirement for written consent as this was a retrospective study. 


\section{Selection of participants}

Databases of the hospital and cardiology department were searched for patients from January 1, 2010, through Dec 31, 2018, who were diagnosed with AF in the ED and then underwent a TEE. The patients' files were then searched to establish the duration of the $\mathrm{AF}$ and whether the patient underwent subsequent cardioversion after negative TEE results during their hospitalization. Patients included in the study were those [?] 18 years of age who presented to the ED with AF > 48 hours' duration and underwent TEE, who had subsequent synchronized electrical cardioversion performed either in the ED or in the cardiology department.

Excluded from the study were those who were: admitted directly to the cardiology ward for cardioversion, hemodynamically unstable, had a clot present on the atrium, with $\mathrm{AF}>48$ hours who underwent pharmacological cardioversion, pregnant, or less than 18 years old.

\section{Data collection and processing}

All data were collected from the hospital's proprietary database and the hospital's cardiology database and entered into an Excel file (Redmond, WA, USA: Microsoft). Data collected included demographic variables (e.g. age, sex), past medical history, chief complaint, date of TEE performance, clinical details of the TEE, date of electrical cardioversion, complications post-cardioversion, and re-admissions within 30 days of hospital discharge. Categorical variables were compared using the Chi-square or Fisher's exact test where appropriate with a $\mathrm{p}<0.05$ considered statistically significant. Continuous variables are described with mean and standard deviation and compared by the Student's t-test where appropriate. The p-values for this are two-tailed, and $\mathrm{p}<0.05$ is considered statistically significant.

\section{Results}

A total of 350 patients diagnosed with AF underwent TEE during the study period. However, after applying the exclusion criteria, the study cohort consisted of 151 patients (43\%). (Figure 1)

Patient and clinical details

Patient gender was almost evenly dispersed; $51 \%(\mathrm{n}=77)$ of patients were male. The mean age was $71+-$ 12.4 years (median $73 ; 27-96)$. Electrical cardioversion was performed in the ED on 92 patients $(61 \%)$ and the cardiology department on 59 (39\%). Most patients' chief complaints were similar between the groups. However, "shortness of breath" was more common in patients cardioverted in the cardiology department ( $\mathrm{p}=0.012)$. Also, "discovering AF as an incidental finding on a routine ECG" was experienced by seven $(8 \%)$ ED patients as opposed to no cardiology department patients $(\mathrm{p}=0.043)$. Demographic details and chief complaints of the cohort comparing those who were cardioverted in the ED versus those converted in the cardiology department are described in Table 1.

Clinical details between the groups were also similar. Vital signs showed no significant differences except for heart rate, which was a mean nine beats per minute higher in those patients cardioverted on the cardiology ward as opposed to those treated in the ED $(\mathrm{p}=0.044)$. Past medical history, as well as lab results, showed no differences between the groups. Troponin is described as "positive" or "negative" rather than as a numerical value since the hospital utilized different methods of troponin measurement during the study period. The term "negative" denotes a negative troponin value for the employed sensitivity test. The highest troponin observed in the entire cohort was a troponin $\mathrm{T}$ of $130 \mathrm{ng} / \mathrm{L}$. This patient was successfully cardioverted in the cardiology department with no adverse effects. (Table 1)

\section{Cardioversion details}

TEE results for the entire cohort showed no thrombus in the left atrium or on the left atrial appendage. Additional echo details are described in Table 2.

The study groups were divided based on where their cardioversion was performed. If cardioversion performed in the ED failed, necessitating hospitalization and re-cardioversion in the cardiology department, these 
patients were classified as "cardiology department" patients (there were two such patients in the study cohort). Most patients from both cohorts had only one cardioversion attempt. "Success of cardioversion" was defined as sinus rhythm at hospital discharge. The great majority (over 90\%) of cardioversions were successful in both groups (95\% in the ED group and $91 \%$ in the cardiology department group). There were very few post-cardioversion complications. However, shortness of breath (SOB) was $10 \%$ more prevalent in those converted on the cardiology ward $(\mathrm{p}=0.030)$, although the absolute numbers were relatively low (two patients in the ED group vs. seven in the cardiology group). Neither chest pain nor hypotension showed any difference between the groups. (Table 2)

\section{Temporal details}

All TEE's were performed within three days of patient arrival, with over $75 \%$ of TEE's performed within the first day. Only seven tests were performed on day three. The time to the performance of TEE was not significantly different between the two groups. The time to cardioversion, however, was significant between the groups. The ED cardioversion group had a markedly shorter time to cardioversion than the cardiology department group, with a mean difference of over 2.5 days longer for the cardiology department patients $(\mathrm{p}<0.001)$. The average time to cardioversion was a mean $1.03+-0.8$ days in the ED group versus $4.17+-$ 1.9 days for the cardiology department group. Correspondingly, the total length of stay (LOS) in the hospital was significantly different between the groups with a mean of $1.49+-1.5$ days for the ED patients versus a nearly five-fold increase of a mean of $7.2+-3.5$ days for the cardiology department patients $(\mathrm{p}<0.001)$. Patients were also kept in the hospital after their cardioversions for significantly different periods of time. The cardiology department cohort was discharged an average of three days after the cardioversion, whereas the ED cohort was discharged less than a day later on average $(\mathrm{p}<0.001)$. (Table 2$)$

\section{Readmissions}

Readmissions (data only from the study hospital) were examined at one-month post-hospital discharge. A total of 32 patients $(21 \%)$ were readmitted to the study hospital one month after hospital discharge. There were 10 fewer readmissions from the cardiology department cohort (11 patients versus 21 patients from the ED cohort), but this difference was not statistically significant $(\mathrm{p}=0.684)$. Causes of readmission were most commonly recurrent $\mathrm{AF}$ in both cohorts (43\% for ED patients versus $46 \%$ for cardiology department patients) but included other causes, both related and unrelated to the primary admission. It was interesting to note that four patients were readmitted to the ED for other cardiac causes (primarily CHF), but no such readmissions reached the cardiology department. No thromboembolic events were reported. Two patients returned due to anticoagulation issues: one due to a high routinely-drawn INR with no reported bleeding and one due to a bleeding event that required no medical intervention. Readmissions occurred after a mean of 11 or 12 days (ED cohort and cardiology department cohort, respectively) and lasted a mean of 3.1 and 3.8 days respectively. (Table 3 )

\section{Discussion}

This is a unique study that compared ED patients with AF for more than 48 hours' duration who subsequently underwent TEE and then electrical cardioversion by emergency physicians back in the ED versus in the cardiology department. Successful cardioversion, defined at discharge, showed that over $90 \%$ of cardioversions were successful in both groups. Time to TEE was approximately one day for both groups. However, time to cardioversion, time kept in the hospital post-procedure, and LOS in the hospital were significantly shorter in the ED group versus the cardiology department group. This was thought to be due to several factors. All patients who were cardioverted underwent procedural sedation. Those who had the procedure performed in the ED had the sedation performed by a second ED physician which could be arranged relatively quickly. However, at the time of the study, the patients who underwent cardioversion in the cardiology department needed to wait for an anesthesiologist to perform the sedation. As there was no dedicated anesthesiologist, this could result in a significant waiting period. Also, there was a logistical need to arrange for a monitored observation area in the cardiology department. Some of the patients in the cardiology department underwent additional workups and treatment for a secondary issue such as anemia. Also, there may have been a trend 
to quickly discharge post-procedure patients from a busy urban ED. Interestingly there was no significant difference in the readmission rate to the hospital one-month post-hospital discharge and no known significant negative outcomes in the ED cardioversion group.

This study is the first to be done to examine ED cardioversion post-TEE performance in patients whose atrial fibrillation was more than 48 hours' duration. Previous studies have described ED electrical cardioversions of less than 48 hours' duration with success rates ranging from 85.5 to $97 \% .{ }^{9}$ Burton et al. described a 7-day $10 \%$ return of patients, of whom $78 \%(25 / 32)$ returned due to a recurrence of AF. ${ }^{1}$ A recent multicenter randomized control trial assigned ED patients with recent-onset $\mathrm{AF}$ ( $<36$ hours) who were symptomatic yet hemodynamically stable to be treated either with early cardioversion or with a "wait-and-see" approach. At 4-week follow-up, AF recurred in 49/164 (30\%) in the delayed cardioversion group versus in 50/171 (29\%) of the earlier cardioversion group. ${ }^{10}$ Scheuermeyer et al. performed a random 30-day follow-up chart review of 400/1233 AF patients who had undergone ED electrical cardioversion. Only 22 patients (5.5\%) returned to the ED within one month for symptoms that were deemed potentially related to the index visit, with six patients $(1.5 \%)$ requiring repeat cardioversion. ${ }^{8}$ The current study described an overall $94 \%$ success rate of rhythm control at discharge (141/151), with an overall 30-day return rate of $22 \%(32 / 151)$, of whom only $44 \%(14 / 32)$ returned due to recurrent $\mathrm{AF}$, constituting $9.3 \%$ of the total study population. It is interesting to note that in the current study, the ED patients fared as well as those in the cardiology cohort. Clinical backgrounds and reasons for ED return also did not differ between the groups. Further investigation into the short-term and longer-term success of ED electrical cardioversion of AF of $>48$ hours' duration after TEE performance is necessary. It seems, based on the results of the current study, that this approach may be at least as successful as some reports of ED cardioversion of AF of $<48$ hours' duration.

It has been noted that patients with $\mathrm{AF}>48$ hours' duration who were treated with standard practice, i.e. weeks of anticoagulation before cardioversion, were not always adequately anticoagulated. ${ }^{11,12}$ TEE studies performed on patients with AF $<48$ hours have shown left atrial thrombi in $1.4 \%$ of patients, of whom $63 \%$ were receiving prior anticoagulation therapy, whereas patients not on prior anticoagulation medication had a $4 \%$ prevalence of left atrial thrombi. ${ }^{13}$ The data from these studies seem to point to the conclusion that timing of onset of AF should not be the only factor in determining a therapeutic approach to AF, and that performance of a TEE to rule out atrial thrombi may be warranted despite prior anticoagulation for even a short duration of AF. In a recent analysis of anticoagulation management with warfarin involving more than 120,000 patients in the Veterans Affairs health care system, the mean proportion of time in the therapeutic range was $58 \%$, with significant variation across sites. ${ }^{14}$ With the new oral anticoagulants, this becomes less of an issue. ${ }^{15}$

The most prominent results of the current study were the differences in length of stay of patients based on where their cardioversion was performed. Time to TEE performance was not significantly different, but once it was performed, time to cardioversion, time to discharge, and inevitably length of hospital stay were all significantly shorter $(\mathrm{p}<0.001$ for the latter three variables) for patients who were cardioverted in the ED versus those cardioverted in the cardiology ward.

Differences in in-patient versus ED lengths of stay in AF patients have previously been shown. Ptaszek et al. demonstrated a marked difference in in-patient LOS after the creation of an AF pathway (64 hours post-intervention versus 104 hours pre-intervention) with no significant corresponding reduction in ED stay. ${ }^{16}$ Other ED studies have cited shorter lengths of stay and higher patient satisfaction with ED AF cardioversion. ${ }^{7,9}$ As the frequency of $\mathrm{AF}$ related hospital visits is predicted to rise, ${ }^{2}$ it is necessary to create strategies to reduce the length of stay, thereby reducing hospital costs. The current study suggests that the performance of TEE in patients with AF of $>48$ hours' duration to rule out thrombi, and subsequent performance of electrical cardioversion in the ED reduces the length of hospital stay and is at least as safe as when performed on the cardiology ward.

Some EDs with fast turn-around time may choose to put such patients into an observation ward. While there is much literature on the use of an ED observation ward to manage patients with atrial fibrillation, none specifically look at the subcategory of those arriving more than 48 hours after onset. ${ }^{17-21}$ For those 
EDs that do have an observation unit, this treatment strategy may be appropriate.

On the other hand, many EDs have problems with crowding resulting in the boarding of patients. ${ }^{22-24}$ This strategy may also be advantageous in this setting, potentially resulting in earlier discharge from the hospital than having the patient wait to be treated on the cardiology ward.

\section{Limitations}

This study had several limitations. This was a retrospective study and there was no recorded reason for the decision as to where to undertake the cardioversion, nor were there data on the reasons to extend patient stay in the cardiology ward. There may have been patient differences not recorded in the data, or it may simply have been a matter of bed allocation.

One of the original study intentions was to collect sedation data along with immediate post-procedure complication data to see if these were related to the sedation or the procedure, but this data was scarce and was therefore ultimately not included in the study. Thus, post-procedure complication data should be cautiously interpreted. Regression analysis was not performed on the data, as most data were similar between the groups.

Many emergency departments may have a policy of automatically admitting patients with AF $>48$ hours to a cardiology ward rather than treating them in the ED or an observation unit. Finally, the study was performed in one tertiary care medical center including a comprehensive cardiac institute, where the practice of ordering a TEE through the ED may not be generalizable to other institutions. Further prospective studies, perhaps of a randomized control design, would be better able to elucidate the safety and efficacy of performing TEE prior to electrical cardioversion of AF > 48 hours' duration in the ED setting.

\section{Conclusion}

In this retrospective, single-center study, patients who presented with atrial fibrillation for more than 48 hours after a TEE negative for an atrial clot, and who were cardioverted in the ED had a significantly decreased time to cardioversion and decreased length of hospital stay when compared to those cardioverted in the cardiological department. There was no significant difference in complications or 30-day readmission rates for either group. This clinical pathway may be implemented in ED observation units. Prospective randomized controlled trials should be conducted to determine the applicability and cost-effectiveness of such a clinical pathway in other ED settings.

\section{References}

1. Burton JH, Vinson DR, Drummond K, Strout TD, Thode HC, McInturff JJ. Electrical cardioversion of emergency department patients with atrial fibrillation. Ann Emerg Med. 2004 Jul;44(1):20-30. doi: 10.1016/j.annemergmed.2004.02.016. Erratum in: Ann Emerg Med. 2004 Sep;44(3):294. PMID: 15226705.

2. Rozen G, Hosseini SM, Kaadan MI, et al. Emergency Department Visits for Atrial Fibrillation in the United States: Trends in Admission Rates and Economic Burden From 2007 to 2014. J Am Heart Assoc. 2018 Jul 20;7(15):e009024. doi: 10.1161/JAHA.118.009024. PMID: 30030215; PMCID: PMC6201465.

3. January CT, Wann LS, Calkins H, et al. 2019 AHA/ACC/HRS Focused Update of the 2014 AHA/ACC/HRS Guideline for the Management of Patients With Atrial Fibrillation: A Report of the American College of Cardiology/American Heart Association Task Force on Clinical Practice Guidelines and the Heart Rhythm Society. J Am Coll Cardiol. 2019 Jul 9;74(1):104-132. doi: 10.1016/j.jacc.2019.01.011. Epub 2019 Jan 28. Erratum in: J Am Coll Cardiol. 2019 Jul 30;74(4):599. PMID: 30703431.

4. Rankin AJ, Rankin SH. Cardioverting acute atrial fibrillation and the risk of thromboembolism: not all patients are created equal . Clin Med (Lond). 2017 Oct;17(5):419-423. doi: 10.7861/clinmedicine.17-5-419. PMID: 28974590; PMCID: PMC6301939.

5. Fried AM, Strout TD, Perron AD. Electrical cardioversion for atrial fibrillation in the emergency department: A large single-center experience. Am J Emerg Med [Internet]. 2020;(xxxx):1-6. Available from: 
https://doi.org/10.1016/j.ajem.2020.02.001

6. Stiell IG, Clement CM, Rowe BH, et al. Outcomes for Emergency Department Patients With Recent-Onset Atrial Fibrillation and Flutter Treated in Canadian Hospitals. Ann Emerg Med [Internet]. 2017;69(5):562571.e2. Available from: http://dx.doi.org/10.1016/j.annemergmed.2016.10.013

7. Jacoby JL, Cesta M, Heller MB, Salen P, Reed J. Synchronized emergency department cardioversion of atrial dysrhythmias saves time, money and resources. J Emerg Med. 2005 Jan;28(1):27-30. doi: 10.1016/j.jemermed.2004.07.011. PMID: 15657000.

8. Xavier Scheuermeyer F, Grafstein E, Stenstrom R, Innes G, Poureslami I, Sighary M. Thirty-day outcomes of emergency department patients undergoing electrical cardioversion for atrial fibrillation or flutter. Acad Emerg Med. 2010 Apr;17(4):408-15. doi: 10.1111/j.1553-2712.2010.00697.x. PMID: 20370780.

9. Cohn BG, Keim SM, Yealy DM. Is emergency department cardioversion of recent-onset atrial fibrillation safe and effective? J Emerg Med [Internet]. 2013;45(1):117-27. Available from: http://dx.doi.org/10.1016/j.jemermed.2013.01.027

10. Pluymaekers NAHA, Dudink EAMP, Luermans JGLM, Meeder JG, Lenderink T, Widdershoven J, et al; RACE 7 ACWAS Investigators. Early or Delayed Cardioversion in Recent-Onset Atrial Fibrillation. N Engl J Med. 2019 Apr 18;380(16):1499-1508. doi: 10.1056/NEJMoa1900353. Epub 2019 Mar 18. PMID: 30883054 .

11. Zaprutko J, Baszko A, Michalak M, et al. Safety of transesophageal echocardiography-guided electrical cardioversion in patients with atrial fibrillation and inadequate anticoagulation. Pol Arch Intern Med. 2017 Aug 9;127(7-8):524-531. doi: 10.20452/pamw.4031. Epub 2017 May 24. PMID: 28817542.

12. Choi EJ, Lee IH, Je NK. Inadequate stroke prevention in Korean atrial fibrillation patients in the post-warfarin era. Int $\mathrm{J}$ Cardiol [Internet]. 2016;220:647-52. Available from: http://dx.doi.org/10.1016/j.ijcard.2016.06.177

13. Kleemann T, Becker T, Strauss M, Schneider S, Seidl K. Prevalence of Left Atrial Thrombus and Dense Spontaneous Echo Contrast in Patients With Short-Term Atrial Fibrillation $<48$ Hours Undergoing Cardioversion: Value of Transesophageal Echocardiography to Guide Cardioversion. J Am Soc Echocardiogr [Internet]. 2009;22(12):1403-8. Available from: http://dx.doi.org/10.1016/j.echo.2009.09.015

14. Rose AJ, Hylek EM, Ozonof A, Ash AS, Reisman JI, Berlowitz DR. Risk-Adjusted percent time in therapeutic range as a quality indicator for outpatient oral anticoagulation results of the veterans affairs study to improve anticoagulation (varia). Circ Cardiovasc Qual Outcomes. 2011;4(1):22-9.

15. Chen ST, Patel MR. Comparison of Anticoagulant Therapy for Atrial Fibrillation - Novel Oral Anticoagulants Versus Vitamin K Antagonists. Prog Cardiovasc Dis. 2018 Jan-Feb;60(4-5):514-523. doi: 10.1016/j.pcad.2018.01.005. Epub 2018 Jan 13. PMID: 29339167.

16. Ptaszek LM, Baugh CW, Lubitz SA, et al. Impact of a Multidisciplinary Treatment Pathway for Atrial Fibrillation in the Emergency Department on Hospital Admissions and Length of Stay: Results of a Multi-Center Study. J Am Heart Assoc. 2019;8(18):e012656.

17. Bellew SD, Bremer ML, Kopecky SL, et al. Impact of an Emergency Department Observation Unit Management Algorithm for Atrial Fibrillation. J Am Heart Assoc. 2016 Feb 8;5(2):e002984. doi: 10.1161/JAHA.115.002984. PMID: 26857070; PMCID: PMC4802469.

18. Conti A, Canuti E, Mariannini Y, et al. Clinical management of atrial fibrillation: Early interventions, observation, and structured follow-up reduce hospitalizations. Am J Emerg Med [Internet]. 2012;30(9):19629. Available from: http://dx.doi.org/10.1016/j.ajem.2012.04.022

19. Cristoni L, Tampieri A, Mucci F, et al. Cardioversion of acute atrial fibrillation in the short observation unit: comparison of a protocol focused on electrical cardioversion with simple antiarrhythmic treatment. 
Emerg Med J. 2011 Nov;28(11):932-7. doi: 10.1136/emj.2009.083196. Epub 2010 Oct 14. PMID: 20947916.

20. Decker WW, Smars PA, Vaidyanathan L, et al. A prospective, randomized trial of an emergency department observation unit for acute onset atrial fibrillation. Ann Emerg Med. 2008 Oct;52(4):322-8. doi: 10.1016/j.annemergmed.2007.12.015. Epub 2008 Mar 14. PMID: 18339449.

21. Koenig BO, Ross MA, Jackson RE. An emergency department observation unit protocol for acute-onset atrial fibrillation is feasible. Ann Emerg Med. 2002;39(4):374-81.

22. Olshaker JS, Rathlev NK. Emergency Department overcrowding and ambulance

diversion: the impact and potential solutions of extended boarding of admitted patients in the Emergency Department. J Emerg Med. 2006 Apr;30(3):351-6. doi: 10.1016/j.jemermed.2005.05.023. PMID: 16677993.

23. Kenny JF, Chang BC, Hemmert KC. Factors Affecting Emergency Department

Crowding. Emerg Med Clin North Am. 2020 Aug;38(3):573-587. doi: 10.1016/j.emc.2020.04.001. Epub 2020 Jun 8. PMID: 32616280.

24. Berg E, Weightman AT, Druga DA. Emergency Department Operations II: Patient

Flow. Emerg Med Clin North Am. 2020 May;38(2):323-337. doi: 10.1016/j.emc.2020.01.002. PMID: 32336328 .

\section{Hosted file}

TEE.Figure1.IJCP.pdf available at https://authorea.com/users/397348/articles/510248-aclinical-pathway-for-safely-and-effectively-cardioverting-emergency-department-patientswith-atrial-fibrillation-greater-than-48-hours

\section{Hosted file}

TEE.Table1.IJCP.pdf available at https://authorea.com/users/397348/articles/510248-aclinical-pathway-for-safely-and-effectively-cardioverting-emergency-department-patientswith-atrial-fibrillation-greater-than-48-hours

\section{Hosted file}

TEE.Table2.IJCP.pdf available at https://authorea.com/users/397348/articles/510248-aclinical-pathway-for-safely-and-effectively-cardioverting-emergency-department-patientswith-atrial-fibrillation-greater-than-48-hours

\section{Hosted file}

TEE.Table3.IJCP.pdf available at https://authorea.com/users/397348/articles/510248-aclinical-pathway-for-safely-and-effectively-cardioverting-emergency-department-patientswith-atrial-fibrillation-greater-than-48-hours 